\title{
Comment on "Surface diffusion near the points corresponding to continuous phase transitions" [J. Chem. Phys. 109, 3197 (1998)]
}

\author{
Vattulainen, Ilpo Tapio; Ying, S. C.; Ala-Nissila, T.; Merikoski, J.
}

Published in:

Journal of Chemical Physics

Link to article, DOI:

$10.1063 / 1.480479$

Publication date:

1999

Document Version

Publisher's PDF, also known as Version of record

Link back to DTU Orbit

Citation (APA):

Vattulainen, I. T., Ying, S. C., Ala-Nissila, T., \& Merikoski, J. (1999). Comment on "Surface diffusion near the points corresponding to continuous phase transitions" [J. Chem. Phys. 109, 3197 (1998)]. Journal of Chemical Physics, 111(24), 11232-11233. https://doi.org/10.1063/1.480479

\section{General rights}

Copyright and moral rights for the publications made accessible in the public portal are retained by the authors and/or other copyright owners and it is a condition of accessing publications that users recognise and abide by the legal requirements associated with these rights.

- Users may download and print one copy of any publication from the public portal for the purpose of private study or research.

- You may not further distribute the material or use it for any profit-making activity or commercial gain

- You may freely distribute the URL identifying the publication in the public portal 


\section{COMMENTS}

\section{Comment on "Surface diffusion near the points corresponding to continuous phase transitions"' [J. Chem. Phys. 109, 3197 (1998)]}

I. Vattulainen

Department of Chemistry, Technical University of Denmark, Building 207, DK-2800 Lyngby, Denmark

S. C. Ying

Department of Physics, Box 1843, Brown University, Providence, Rhode Island 02912-1843

T. Ala-Nissila

Department of Physics, Box 1843, Brown University, Providence, Rhode Island 02912-1843, Helsinki Institute of Physics, P.O. Box 9 (Siltavuorenpenger 20 C), FIN-00014 University of Helsinki, Finland and Laboratory of Physics, Helsinki University of Technology, P.O. Box 1100, FIN-02015 HUT, Finland

J. Merikoski

Department of Physics, University of Jyväskylä, P.O. Box 35, FIN-40351 Jyväskylä, Finland

(Received 17 February 1999; accepted 1 October 1999)

[S0021-9606(99)52148-X]

In a recent article, ${ }^{1}$ Uebing and Zhdanov (UZ) reported results of Monte Carlo (MC) simulations for diffusion of oxygen adatoms on the $\mathrm{W}(110)$ surface close to a secondorder phase transition boundary. It is well known ${ }^{2}$ that unlike static equilibrium properties, kinetic quantities in MC are very sensitive to the details of the algorithm used for the microscopic transition rates between different configurations, and one would expect an even larger sensitivity in the critical region. We show in this Comment that this is indeed the case. Our results imply that the particular dynamic algorithm used by UZ (Ref. 1) is inappropriate for studies of critical effects in diffusion. As a consequence, their conclusion on the critical effects of diffusion coefficients is incorrect and the criticism presented by UZ concerning our previous work ${ }^{3}$ is unjustified.

We start by assessing the validity of various dynamical algorithms, including the one chosen by UZ, in describing the diffusion process in terms of MC simulations. To this end, we consider a model lattice-gas Hamiltonian for the $\mathrm{O} / \mathrm{W}(110)$ system. ${ }^{3,4}$ Unlike the one used by UZ, it produces a phase diagram in close agreement with the experimental observations for $\mathrm{O} / \mathrm{W}(110),{ }^{5}$ including all known ordered phases and coexistence regions. To introduce the dynamics in $\mathrm{MC}$, we consider the following choices for the transition rate $w_{i, f}$ from an initial state $i$ with energy $\epsilon_{i}$ to a final state $f$ with energy $\epsilon_{f}$ :

(1) The Metropolis form, ${ }^{2}$ in which $w_{i, f}=\exp \left[-\left(\epsilon_{f}\right.\right.$ $\left.\left.-\epsilon_{i}\right) / k_{B} T\right]$, if $\epsilon_{f}>\epsilon_{i}$. Otherwise $w_{i, f}=1$.

(2) The Kawasaki form, ${ }^{2}$ in which $w_{i, f}=1 /\left(1+\exp \left[\left(\epsilon_{f}\right.\right.\right.$ $\left.\left.-\epsilon_{i}\right) / k_{B} T\right]$ ).

(3) The transition dynamics algorithm (TDA), ${ }^{6}$ where the transition proceeds by two steps via an intermediate state $I$ with energy $\epsilon_{I}=\Delta+\left(\epsilon_{i}+\epsilon_{f}\right) / 2$ such that $w_{i, f}$ $=w_{i, I} w_{I, f}$. The rates for the two steps are of the Me- tropolis form and the quantity $\Delta>0$ characterizes the effect of the saddle point of the adiabatic substrate potential.

(4) The dynamics of UZ, ${ }^{1}$ in which $w_{i, f}=(1 / \kappa) \exp \left[-\left(\epsilon_{S}\right.\right.$ $\left.\left.-\epsilon_{i}\right) / k_{B} T\right]$ and $\kappa$ is a normalization constant. The quantity $\epsilon_{S}=\epsilon_{S}^{0}+\phi_{N}^{*} \Sigma_{N N} c_{j}^{\prime}$ is chosen to model the saddle point energy, and $\epsilon_{S}^{0}$ is the corresponding quantity in the limit of low coverages $\theta \rightarrow 0$. The value of $\epsilon_{S}^{0}$ used has not been reported, ${ }^{1,7}$ but our choice $\epsilon_{S}^{0}=0$ does not affect our conclusions. The term $\Sigma_{N N} c_{j}^{\prime}$ is a sum of the occupation variables of "nearest neighbors" with respect to the transition state, and $\phi_{N}^{*}$ is an interaction parameter.

In Fig. 1(a) we show our simulation results for the average single-particle transition rate $\Gamma \equiv\left\langle n_{i}\left(1-n_{f}\right) w_{i, f}\right\rangle$ at $\theta$ $=0.45$ around the second-order phase transition boundary with the algorithm choices (1)-(4) described above $\left(n_{i}\right.$ and $n_{f}$ are the occupation variables of the initial and final sites, respectively). Clearly, the results of the dynamics (4) used by UZ are distinctly different from those of (1)-(3). The difference is further demonstrated by considering the effective activation barriers $E_{A}(\theta, T) \equiv-\partial \ln \Gamma(\theta, T) / \partial\left(1 / k_{B} T\right)$ shown in Fig. 1(b). The dynamics of (1)-(3) show a prominent peak near $T_{c}$, whereas the dynamics of UZ shows a much smaller cusp. ${ }^{8}$

The physical origin of the discrepancy between the results using the UZ algorithm and those from the other three algorithms stems from the choice of saddle point energies in terms of the interaction parameter $\phi_{N}^{*}$. The choice of $\phi_{N}^{*}$ is completely arbitrary and cannot be judged by experimental data. Further, since it is not related to the true interaction parameters in the Hamiltonian, the influence of the critical fluctuations near $T_{c}$ to the saddle point configurations and to the diffusion coefficient is largely left out. To demonstrate this, we can consider the special case of the UZ algorithm 


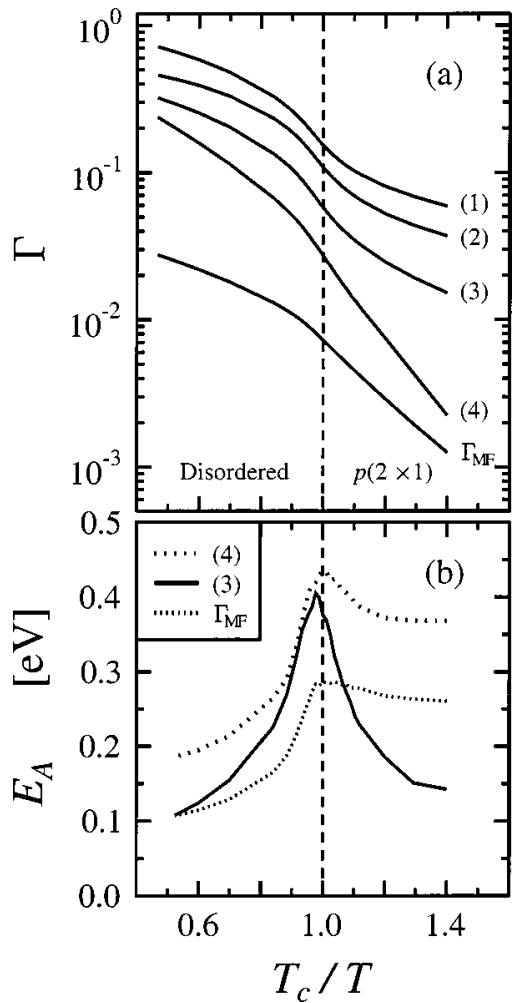

FIG. 1. (a) $\Gamma$ for various dynamical algorithms in the $\mathrm{O} / \mathrm{W}(110)$ system (Refs. 4, 3) with $\Gamma_{\mathrm{MF}}$ also shown (some curves have been shifted for clarity). In the dynamics of UZ, we chose $\phi_{N}^{*}$ as the pair interaction between second nearest neighbors (Ref. 1). In TDA, $\Delta=0.044 \mathrm{eV}$ (larger values give similar results). (b) Effective activation barriers $E_{A}$ corresponding to the rates shown in (a). Results of items (1) and (2) are not shown, since they are almost identical to those of item (3).

with the choice $\phi_{N}^{*}=\epsilon_{S}^{0}=0$. In this case, the transition rate is only determined by the initial configuration and can be represented as ${ }^{1}$

$$
\Gamma_{\mathrm{MF}}=\Gamma_{0}\left(\mathcal{P}_{00} / \theta\right) \exp \left(\mu / k_{B} T\right) .
$$

Here $\mathcal{P}_{00}$ is the probability that a nearest neighbor pair is vacant, $\mu$ is the chemical potential, and $\Gamma_{0}(T)$ is the bare jump rate (in the limit $\theta \rightarrow 0) .{ }^{1}$ Aside from the constant $\Gamma_{0}(T), \Gamma_{\mathrm{MF}}$ contains only static quantities and no dynamical information at all. It is obviously a mean-field-type of description for the true transition rate. In Fig. 1(a) we plot $\Gamma_{M F}$ as computed for the present model. It is qualitatively very similar to the result from using the UZ algorithm with finite $\phi_{N}^{*} .{ }^{9}$ They both fail to describe the strong non-Arrhenius behavior of $\Gamma$ near $T_{c}$ clearly revealed by the choices (1)-(3). ${ }^{10}$

To further demonstrate the unrealistic choice of the saddle point energy in the UZ algorithm, we have performed molecular dynamics simulations using effective medium theory for some fcc metal systems ${ }^{11}$ and mapped the interactions to a lattice-gas description. We calculated the true ac- tivation barrier for every configuration change and compared the energetics of the saddle point configuration to the form postulated in the UZ dynamics to estimate $\phi_{N}^{*}$. It turns out that the values of $\phi_{N}^{*}$ obtained in this fashion vary over a wide range of values, having both positive (repulsive interactions) and negative (attractive) values. This inconsistency of the UZ dynamics is in contrast with, e.g., the TDA dynamics, ${ }^{6}$ which describes the instantaneous jumps via a transition state in a simple but physically sensible manner.

To conclude, the present results demonstrate the sensitivity of activated rates close to $T_{c}$ to the choice of the dynamical algorithm in MC simulations. This observation is of general concern and necessitates further work to clarify the reasons of why various commonly used algorithms lead to distinctly different behavior of kinetic quantities in MC studies. In the present case, we have shown that the dynamics used by UZ suppress an essential part of the physical problem under study, namely, the effect of the critical fluctuations on the microscopic transition rates $\Gamma$ close to $T_{c} \cdot{ }^{12} \mathrm{~A}$ true description of the critical fluctuations around both the equilibrium configurations and the transition states is therefore crucial for a correct MC study of the critical dynamics effect. One realistic approach to accomplish this effect is the TDA dynamics discussed above. Nevertheless, in spite of the long history of MC, it is clear that this issue still requires more attention to clarify the role of dynamical algorithms in general.

${ }^{1}$ C. Uebing and V. P. Zhdanov, J. Chem. Phys. 109, 3197 (1998).

${ }^{2}$ See K. W. Kehr and K. Binder, in Applications of the Monte Carlo Method in Statistical Physics, edited by K. Binder (Springer-Verlag, Berlin, 1984), and references therein.

${ }^{3}$ I. Vattulainen, J. Merikoski, T. Ala-Nissila, and S. C. Ying, Phys. Rev. B 57, 1896 (1998); Phys. Rev. Lett. 79, 257 (1997).

${ }^{4}$ I. Vattulainen, S. C. Ying, T. Ala-Nissila, and J. Merikoski, Phys. Rev. B 59, 7697 (1999).

${ }^{5}$ G.-C. Wang, T.-M. Lu, and M. G. Lagally, J. Chem. Phys. 69, 479 (1978); C. R. Brundle and J. Q. Broughton, in The Chemical Physics of Solid Surfaces and Heterogeneous Catalysis: Chemisorption Systems, edited by D. A. King and D. P. Woodruff (Elsevier, Amsterdam, 1990), Chap. 3, Vol. 3A; K. E. Johnson, R. J. Wilson, and S. Chiang, Phys. Rev. Lett. 71, 1055 (1993).

${ }^{6}$ T. Ala-Nissila, J. Kjoll, and S. C. Ying, Phys. Rev. B 46, 846 (1992).

${ }^{7}$ C. Uebing and R. Gomer, Surf. Sci. 381, 33 (1997).

${ }^{8}$ Close to a critical point where fluctuations are enhanced, there is a wellknown "compensation effect" for barriers of activated processes. This is due to the entropic contribution to the rate coefficients. Thus, a peak for $E_{A}$ at $T_{c}$ is to be expected.

${ }^{9}$ In Ref. 1 , results for the case where $\phi_{N}^{*}=0$ and where it is nonzero show similar behavior around $T_{c}$.

${ }^{10} \mathrm{In}$ fact, in Ref. $1 \mathrm{UZ}$ finds a broad peak in the activation barriers around $1.2 T_{c}$, instead of $T_{c}$ as expected (Ref. 8).

${ }^{11}$ J. Merikoski, I. Vattulainen, J. Heinonen, and T. Ala-Nissila, Surf. Sci. 387, 167 (1997).

${ }^{12}$ The criticism in Ref. 1 by UZ concerning finite-size effects in our works is irrelevant, and fully discussed and refuted in our work [I. Vattulainen, J. Merikoski, T. Ala-Nissila, and S. C. Ying, Phys. Rev. Lett. 80, 5456 (1998)]. 